\title{
The tomato cytosolic fructokinase FRK1 is important for phloem fiber development
}

\author{
O. STEIN ${ }^{1,2}$, F. SECCHI ${ }^{3}$, M.A. GERMAN ${ }^{1}$, H. DAMARI-WEISSLER ${ }^{1}$, R. ALONI ${ }^{4}$, N.M. HOLBROOK ${ }^{5}$, \\ M.A. ZWIENIECKY ${ }^{6}$, and D. GRANOT ${ }^{1 *}$
}

Institute of Plant Sciences, Agricultural Research Organization, The Volcani Center, 50250, Bet Dagan, Israel ${ }^{1}$ The Institute of Plant Sciences and Genetics in Agriculture, The Hebrew University of Jerusalem, 76100, Rehovot, Israel ${ }^{2}$

Department of Agricultural, Forest and Food Sciences, University of Torino, 10095, Grugliasco, TO, Italy ${ }^{3}$ Department of Molecular Biology and Ecology of Plants, Tel Aviv University, 69978, Tel Aviv, Israel ${ }^{4}$ Department of Organismic and Evolutionary Biology, Harvard University, Cambridge, 02138, MA, USA Department of Plant Sciences, University of California, Davis, 95616, CA, USA ${ }^{6}$

\begin{abstract}
Tomato (Solanum lycopersicum) plants have four fructokinase genes, SlFRK1-4. The SlFRK4 is expressed only in pollen, whereas the other three are expressed in all plant parts. While SlFRK2 and SlFRK3 are involved in vascular tissue development and affects the shape, size, and cell-wall width of xylem vessels and xylem fibers, the role of SlFRK1 has not been studied previously. The current work investigates the expression of SlFRK1 using transgenic tomato plants expressing the $\beta$-glucuronidase reporter gene under the SlFRK1 promoter, as well as the role of SlFRK1 using transgenic plants with antisense suppression of SlFRK1. The SlFRK1 promoter was expressed primarily in vascular tissues and specific suppression of SIFRK1 reduced water transport in stems, but had no other anatomical or phenotypic effects. Combined suppression of SlFRK1 and SlFRK2 severely inhibited plant growth and an anatomical analysis revealed a reduction in secondary xylem area and distorted phloem fibers characterized by thin cell walls and reduced lignification. The results suggest that SIFRK1 is involved in vascular tissue development and hydraulic conductivity in tomato plants and that SIFRK1 is important for normal phloem fiber development together with SlFRK2.
\end{abstract}

Additional key words: cell walls, hydraulic conductivity, lignification, water transport, xylem vessels.

\section{Introduction}

Sucrose, a disaccharide, is an important end product of photosynthesis and a primary carbon source for metabolism in sink tissues of many plants including tomato (Solanum lycopersicum). Sucrose must be cleaved by sucrose synthase (SUS), into UDP-glucose and fructose, or by invertase into glucose and fructose to be further metabolized (Dennis and Blakeley 2000). The free hexoses, fructose and glucose, must then be phosphorylated by fructokinase (FRK) or hexokinase (HXK) before they can enter metabolic pathways. The FRK and HXK are distinguished by their substrate specificities and affinities (Renz and Stitt 1993, Dai et al. 2002, Granot 2007). The FRK phosphorylates only fructose; whereas HXK phosphorylates both glucose and

fructose. However, the affinity of FRK for fructose is two orders of magnitude greater than that of HXK to fructose. It is, therefore, likely that fructose is phosphorylated primarily by FRK (Granot 2007).

The FRK genes have been identified in many plant species including tomato, potato, maize, soybean, barley, spinach, and pea. In tomato, four FRK genes (SlFRK1-4) have been identified. They encode enzymes with different intracellular localization and biochemical characteristics. The tomato FRKs are expressed at different levels in almost all plant parts with the exception of SlFRK4, which is expressed specifically in stamens and pollen (German et al. 2002, 2003, 2004, David-Schwartz et al. 2013, Granot et al. 2013). Three of the tomato FRK

Submitted 17 February 2017, last revision 18 May 2017, accepted 12 June 2016.

Abbreviations: FRK - fructokinase; GUS - $\beta$-glucuronidase; HXK - hexokinase; Kh - hydraulic conductivity; SUS - sucrose synthase.

* Corresponding author; fax: (+972) 3 9669642, e-mail: granot@agri.gov.il 
enzymes are found in the cytosol and a single FRK (SlFRK3) is found in plastids (Damari-Weissler et al. 2006).

Some FRK enzymes are inhibited by their own substrate, fructose, when the fructose concentration exceeds a certain level, usually $1 \mathrm{mM}$, a phenomenon known as substrate inhibition (Gardner et al. 1992, Renz and Stitt 1993, Dai et al. 1997). In tomato, two FRK isozymes, SIFRK2 and SIFRK3, are substrate inhibited, whereas SIFRK1 and SIFRK4 are not (Petreikov et al. 2001, German et al. 2002, 2004). In addition, the affinity of SIFRK1 for fructose is about 20-fold less than that of SIFRK2 and SIFRK3 and only 2- to 8-fold greater than the affinities of the four tomato HXKs, SlHXK1-4 (Granot 2007).

The different characteristics of the various FRKs suggest that they may play different roles. Increasing evidence suggests that FRKs are important for vascular development. The tomato SlFRK2 is essential for proper vascular development as stems of SlFRK2-antisense plants have xylem vessels and xylem fibers with thinner secondary cell walls and the xylem vessels of those plants are also narrower and deformed (Damari-Weissler et al. 2009). As a result, water transport is reduced, causing severe growth inhibition and the wilting of young leaves (Damari-Weissler et al. 2009). The tomato plastidic FRK, SlFRK3, is also important for xylem development. RNAi

\section{Materials and methods}

Plants and sequencing: Tomato (Solanum lycopersicum Mill.) plants of cultivar MP1 were used to generate all of the transgenic lines. The DNA of the BAC-167A6 was used for PCR amplification of the SIFRK1 promoter region. A 1673-bp fragment of the $S I F R K 1$ promoter was amplified with a pair of primers carrying EcoRI and a BamHI restriction sites. After digestion, the DNA fragment was inserted into the $\mathrm{pE} 234$ plant binary vector, which had been predigested with EcoRI and a BamHI. The pE234 includes a GUS reporter gene and the neomycin phosphotransferase II (NptII) gene as a selectable marker.

The partial sequence between 880 and the $3^{\prime}$ end of the FRK1 cDNA (Kanayama et al. 1997) was amplified using PCR. The primer contained an engineered SacI site (5'-CTAGCGAGCTCGAGTGGAATAATGAG-3'). The resulting 700-bp fragment was digested with SacI and BamHI $(+1580)$. Each fragment was inserted between the cauliflower mosaic virus $35 \mathrm{~S}$ promoter and nopalinesynthase termination site in the binary vector pBI121 (Clontech, Palo Alto, CA, USA), in which the GUS sequence was removed by digesting with BamHI (Odanaka et al. 2002). Both constructs were transformed into cv. MP-1 via Agrobacterium-mediated transformation. The PCR was used to detect the presence of the nptII gene among independent $\mathrm{T}_{0}$ transgenic plants. Homozygous individuals were identified among $T_{1}$ seeds following kanamycin-resistant segregation of nptII. suppression of SlFRK3 decreased plant hydraulic conductivity and transpiration, but had no visual effect on plant growth. Yet, combined suppression of both the cytosolic SIFRK2 and the plastidic SIFRK3 disturbed not only xylem vessels, but also the development of xylem fibers, resulting in unlignified and distorted xylem fibers (Stein et al. 2016). FRK is also important for the development of xylem fibers in aspen (Populus tremula $\times$ tremuloides), in which the suppression of the cytosolic FRK2 yielded narrower xylem fibers probably due to decrease in cellulose deposition (Roach et al. 2012). FRKs have also been found to be important for vascular tissue development in Arabidopsis. Arabidopsis plants harboring mutations in several FRKs (together) showed reduced xylem area and necrotic lesions in their xylem, cambium and the phloem of their hypocotyls, which led to reduced hydraulic conductivity and wilting of leaves and, eventually to plant death (Stein et al. 2017).

Of the three tomato FRKs expressed in various tissues, the role of SIFRK1 has been the least studied. In a previous study, suppression of SIFRK1 delayed flowering with no other effects on plant growth (Odanaka et al. 2002). The aim of this work was to explore the expression pattern and function of SlFRK1 and to examine whether this gene also plays a role in vascular development.

Histochemical localization of $\beta$-glucuronidase activity: To localize the GUS activity, leaves, stems, petioles, fruits, and flowers of the transgenic plants were infiltrated with $1 \mathrm{mM}$ 5-bromo-4-chloro-3-indolyl-glucuronide (XGluc) in $50 \mathrm{mM}$ sodium phosphate buffer (pH 7) containing $0.5 \mathrm{mM} \mathrm{K}{ }_{3} \mathrm{Fe}(\mathrm{SCN})_{6}, 0.5 \mathrm{mM} \mathrm{K}_{4} \mathrm{Fe}(\mathrm{SCN})_{6}$, and $1 \mathrm{mM}$ EDTA and incubated at $37{ }^{\circ} \mathrm{C}$ overnight. After incubation, the plant material was cleared with $70 \%(\mathrm{v} / \mathrm{v})$ ethanol, and the leaves were photographed. Free-hand cross-sections were made from petioles and stems to visualize GUS activity at the cellular level. These crosssections were rinsed in water and examined under transmitted white light. Fruits, leaves, and flowers were photographed under a dissecting microscope.

RNA extraction and cDNA generation: Leaf samples from tomato plants were frozen and homogenized in liquid nitrogen. RNA was extracted using the EZ-RNA kit (Biological Industries, Kibbutz Bet Haemek, Israel) with up to $0.5 \mathrm{~cm}^{3}$ of frozen homogenized tissue per extraction tube. Two to three independent extractions were performed for each tissue set. The extractions were carried out according to the manufacturer's instructions. RNA pellets were suspended in $0.025 \mathrm{~cm}^{3}$ of DEPCtreated $\mathrm{H}_{2} \mathrm{O}$ and treated with DNase (Ambion, Austin, TX, USA) according to the manufacturer's instructions. The presence of RNA was confirmed by gel electrophoresis and DNA degradation was confirmed by PCR. 
The RNA $(\leq 1 \mu \mathrm{g})$ from each sample was reversetranscribed to cDNA using MMLV RT kit (Promega, Madison, WI, USA) in a $25-\mathrm{mm}^{3}$ reaction tube, with $2 \mathrm{~mm}^{3}$ of random primers and $1 \mathrm{~mm}^{3}$ of mixed poly-dT primers (18 to $23 \mathrm{nt}$ ) for the generation of cDNA from both rRNA and mRNA. All cDNA samples were diluted $1: 8$ with water.

Real-time expression analysis: Real-time reactions were prepared with SYBR Green mix (Eurogentec, Seraing, Belgium) in $10-\mathrm{mm}^{3}$ aliquots with $4 \mathrm{~mm}^{3}$ of diluted cDNA per reaction, two replicates per cDNA sample. Reactions were run in a RotorGene 6000 cycler (Corbett, Mortlake, Australia), 40 cycles per run, with sampling after each cycle. Results were interpreted with RotorGene software and normalized for each tissue set (three independent samples, two duplicates per sample). Cyclophilin was used as a reference gene for normalization.

Hydroponic cultivation: Seeds were planted on wet filter paper (Whatman ${ }^{\circledR}$, Schleicher \& Schuell, Dassel, Germany) in covered Petri dishes and left to germinate in the dark at room temperature. Three days after germination, the seedlings were moved to aerated hydroponic culture in $6.5-\mathrm{dm}^{3}$ containers filled with modified Hoagland solution (Hoagland and Arnon 1950, Damari-Weissler et al. 2009) and maintained in a growth chamber (an irradiance of $600 \mu \mathrm{mol} \mathrm{m} \mathrm{m}^{-2} \mathrm{~s}^{-1}$, a 14-h photoperiod, day/night temperatures $25 / 21{ }^{\circ} \mathrm{C}$, and an air humidity of $65 \%$ ). After one week, the young plants were transferred to $42-\mathrm{dm}^{3}$ boxes ( 12 plants per box) and the culture media were replaced twice a week.

Forced root exudation: The hydraulic conductance of tomato root systems was determined by measuring the flow induced in response to an applied pressure gradient (Gorska et al. 2008). De-topped root systems were fitted with a plastic tube filled with distilled water and connected to a beaker located on a balance $( \pm 0.01 \mathrm{mg})$. The root system was sealed in a chamber containing the hydroponic solution in which the plants had been grown. The $\mathrm{pH}$ of the solution was kept at $\sim 6.1$ using 2-(N-morpholino) ethane sulfonic acid (MES) buffer $\left(1 \mathrm{~g} \mathrm{dm}^{-3}\right)$. The pressure in the chamber was regulated using a needle valve, which was adjusted to allow a small leak in the chamber, such that air used to pressurize the chamber also served to aerate the medium. Water flow through the root system was automatically recorded by a computer at $30 \mathrm{~s}$ intervals. Flow stabilization occurred
10 to $20 \mathrm{~min}$ after the plant was exposed to pressure. Flow data were then collected for $1 \mathrm{~h}$. At the end of each experiment, the fresh mass of the roots was recorded. The roots were then dried in an oven at $90{ }^{\circ} \mathrm{C}$ for $72 \mathrm{~h}$, after which the dry mass was recorded and then root flow was calculated according to Gorska et al. (2008).

Stem hydraulic conductivity: Small sections of stems $(\sim 5 \mathrm{~cm}$ long) were cut under water to prevent embolisms caused by air entering into the cut vessel. In addition, stems were perfused under elevated pressure $(150 \mathrm{kPa})$ for few minutes to remove pre-existing embolism. The hydraulic conductivity $(K)$ of each stem segment was measured gravimetrically by determination of flow rate of filtered and degassed $10 \mathrm{mM} \mathrm{KCl}$ solution. A solution was located on a balance (Sartorius; precision $\pm 0.1 \mathrm{mg}$ ) and connected to the stem by a plastic tube. The stem was submerged in a water bath with the water level being $\sim 10 \mathrm{~cm}$ below the level of water on the balance. After a steady flow rate was reached (within just a few minutes), the specific hydraulic conductivity $(\mathrm{Kh})$ was calculated as volumetric flow rate $\left[\mathrm{kg} \mathrm{s}^{-1}\right]$ divided by the pressure difference across the stem length $\left[\mathrm{MPa} \mathrm{m}^{-1}\right]$. Kh was then normalized either by xylem cross-sectional area $\left[\mathrm{m}^{2}\right]$ or leaf area $\left[\mathrm{m}^{2}\right]$ to calculate either $\mathrm{Ks}$ (xylem specific conductivity) or $\mathrm{Kl}$ (leaf specific conductivity) $\left[\begin{array}{lll}\mathrm{kg} & \mathrm{s}^{-1} & \mathrm{~m}^{-1} \\ \mathrm{MPa}^{-1}\end{array}\right]$. Xylem cross-sectional area was microscopically determined for each stem. Leaf area was determined for all leaves above the cut section using digitized leaves images.

Anatomical techniques: To analyze the anatomy of all of the plants, stem samples were taken from at least two independent plants from each line at the same time and stored in $70 \%(\mathrm{v} / \mathrm{v})$ ethanol until examination. Free-hand cross-sections were taken, stained for a few seconds in $2 \%(\mathrm{~m} / \mathrm{v})$ lacmoid (Sigma-Aldrich, St. Louis, MO, USA) in $96 \%(\mathrm{v} / \mathrm{v})$ ethanol and then rinsed in tap water for a few minutes, mounted in $50 \%(\mathrm{~m} / \mathrm{v})$ sodium lactate (Aloni 1980), and observed under transmitted white light. The lacmoid stained (marine blue) the lignin in the cell walls of the vessels and the fibers. Cross-sections were also stained with phloroglucinol and mounted in phloroglucinol-HCl for specific lignin staining (Ruzin 1999).

Statistical analysis was performed using the JMP 5.0 software program. Means were compared using Student's $t$-test.

leaves. Thorough promoter expression analysis revealed that the SIFRK1 promoter was expressed in stamens and in vascular tissues of leaves, petioles, stem, roots, and fruits, (Fig. 1). The SlFRK1 promoter, similar to the SlFRK2 and SlFRK3 promoters, was expressed primarily in the stem secondary xylem (Fig. $1 E$ ). Yet, while the
To determine in which tissues SlFRK1 is expressed, we isolated a $1.7 \mathrm{~Kb}$ fragment of the SlFRK3 promoter region including the 5 , UTR, fused it to the $\beta$-glucuronidase $(G U S)$ reporter gene and used it to generate transgenic tomato plants. Three independent transgenic lines showed GUS staining in the vascular tissues of their 
SlFRK2 promoter is expressed throughout the entire secondary xylem (Fig. 1E Suppl.) and the SlFRK3 promoter was expressed mainly in the cambium and newly differentiating xylem cells (Fig. $2 C$ Suppl.), the SIFRK1 promoter was expressed primarily in the mature secondary xylem and not in the cambium or differentiating xylem (Fig. 1E). In addition, the SlFRK1 promoter was expressed in phloem companion cells of both internal and external phloem (Fig. 1C,F). This was different from SlFRK2 and SlFRK3, whose promoters were expressed in either internal or external phloem, respectively (Figs. $1 F, 2$ Suppl.).
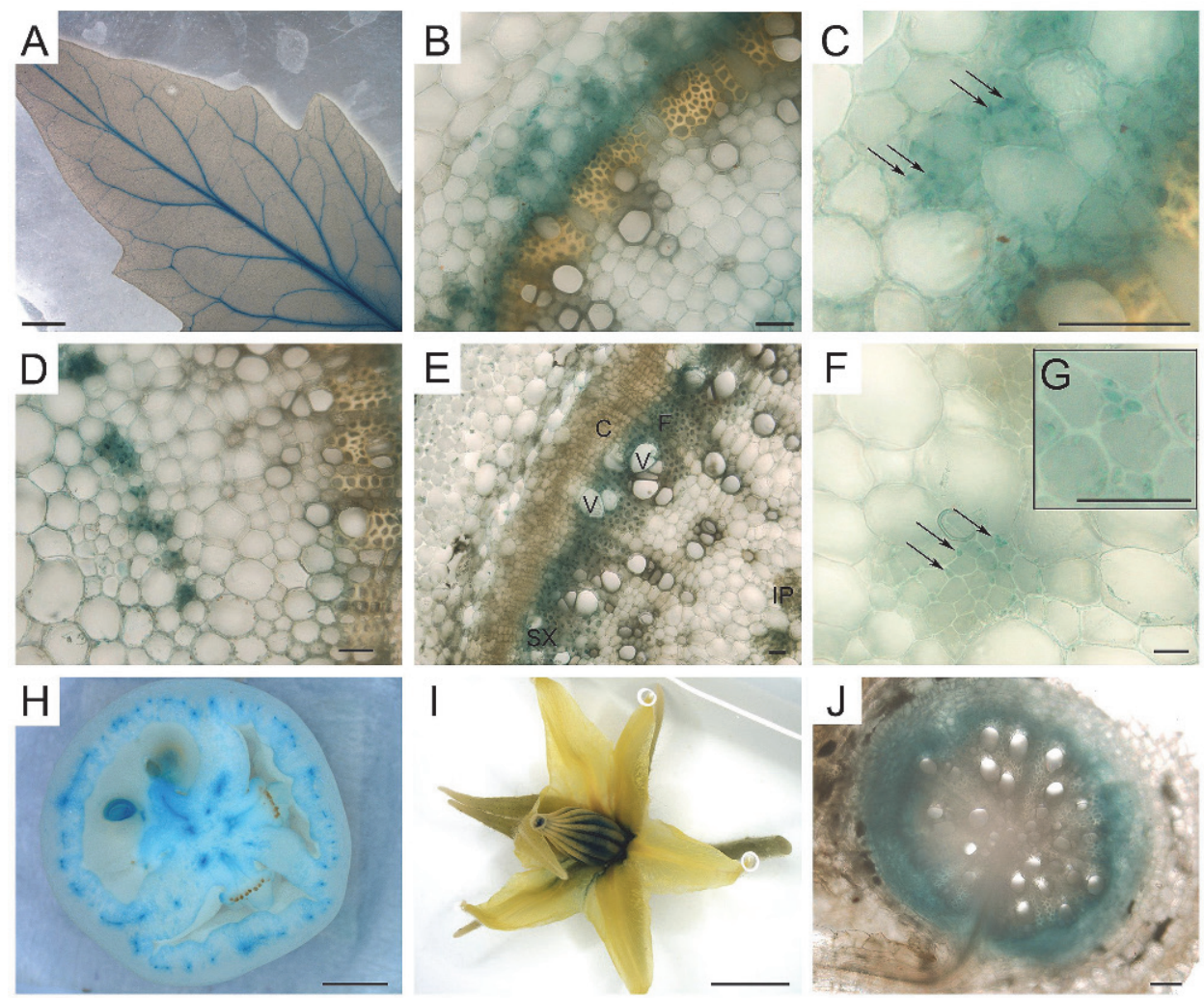

Fig. 1. Histochemical analysis of the expression pattern of the SIFRK1 promoter visualized by GUS staining in tomato tissues: $A$ - leaf, $B, C$ - cross-sections of leaf petiole, $D$ - $G$ - stem cross-sections, $H$ - young green fruit, $I$ - opened flower, and $J$ - primary root (C - cambium, F - xylem fibers, V - vessel, SX - secondary xylem, IP - internal phloem; black arrows - phloem companion cells; scale bars: $A, H, I-5 \mathrm{~mm}, B-G, J-50 \mu \mathrm{m})$.

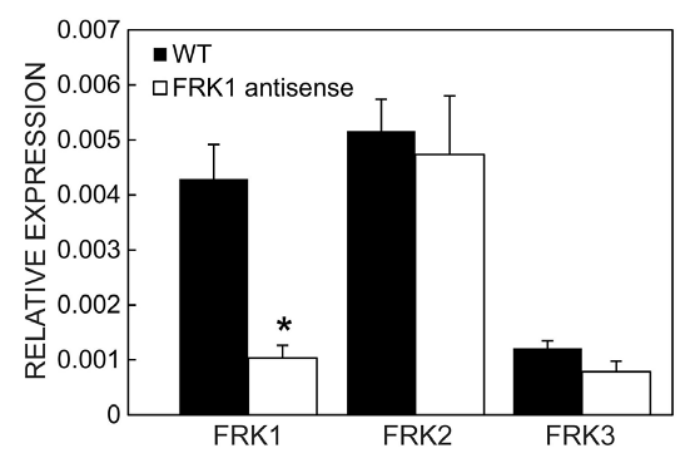

Fig. 2. Expression of SlFRK genes in leaves of the FRK1antisense line. Samples were taken from leaf 4. Relative expression of three SlFRK genes in the FRK1-antisense line and WT plants was determined by real-time expression analysis with gene-specific primers (German 2003). Expression was normalized to the expression of cyclophylin in each sample. Means \pm SEs, $n=3$, * a statistically significant difference $(t$-test; $P<0.05)$.
The FRK-promoter expression patterns in the vascular tissues (Fig. 1) correlate well with fructosephosphorylation activity, which was found to be 2- to 3 -times higher in the secondary xylem than in the pith or bark (German et al. 2003). The complex expression patterns of tomato FRKs together with the specific roles of FRK2 in the development of tracheary elements (Damari-Weissler et al. 2009) and FRK3 and FRK2 in xylem fiber development (Stein et al. 2016) suggest that FRK1 may also play a specific and unique role in vascular development.

Fructose phosphorylation may be crucial for fructose metabolism in various non-photosynthetic cells and we might expect to detect expression of at least one FRK isozyme in other sink cells, such as the cells of the root cortex. One possible reason for the lack of GUS expression in other types of cells is that HXKs are sufficient for fructose phosphorylation, despite their relatively low affinity for fructose. But, it is also possible that the $1.5-2 \mathrm{~kb}$ promoter fragments used for the tomato 
FRK gene expression analyses might not include all the regulatory elements responsible for gene expression. A good example of that type of scenario is the tomato sucrose transporter 1 (LeSUT1), in which some of the regulatory sequences promoting expression in phloem companion cells, trichomes, and guard cells are found in introns (Weise et al. 2008). Nevertheless, the analyses of GUS expression provide a strong indication of the putative roles of FRKs in vascular tissues.

To study the role of SIFRK1 in planta, we generated transgenic tomato plants with an antisense fragment of SlFRK1 expressed under the $35 \mathrm{~S}$ promoter. Seven independent lines were created and confirmed as transgenic by PCR. Homozygous plants were identified among $T_{1}$ plants using kanamycin-resistance segregation analysis. Out of the seven homozygous lines, only one line, a84, showed reduced expression of the SIFRK1 gene (Fig. 3 Suppl.). The a84 transgenic line, which will be referred to from now on as the FRK1-antisense line, showed specific FRK1 suppression, with about $77 \%$ reduction in SlFRK1 expression, but no effects on the expression of SlFRK2 or SlFRK3 (Fig. 2).

Under normal growing conditions, the FRK1antisense plants did not show any altered phenotype, relative to WT plants. However, FRK1-antisense plants had reduced water conductance in their stems (Fig. 3A,B), but not in their roots (Fig. $3 C$ ). This effect is different from that observed in plants with specific suppression of either FRK2 or FRK3, in which both stem and root hydraulic conductivity were reduced (Damari-Weissler et al. 2009, Stein et al. 2016). Although all three promoters were found to be expressed in the vascular tissue of roots, real-time PCR results showed that SlFRK1 expression in roots is very low compared to that of SlFRK2 and SlFRK3 (German et al. 2004). This may be the reason why FRK1 suppression had no effect on root hydraulic conductivity. These results may indicate that FRK1 plays a primary role in stems. Yet, unlike suppression of FRK2, which has significant anatomical effects on the stem vascular tissues (Damari-Weissler et al. 2009), no clear anatomical effects were observed in FRK1-antisense stems (Fig. 5B).

In a previous study, we demonstrated that specific suppression of FRK3 also reduced stem hydraulic conductivity with no effects on vascular anatomy, but combined suppression of FRK2 and FRK3 had very significant anatomical effects, indicating that FRK2 and FRK3 may compensate for one another in vascular development. To explore compensation between FRK1 and FRK2 or FRK3, we crossed FRK1-antisense plants with either FRK2-antisense or FRK3-RNAi plants that had specific suppression of FRK2 or FRK3, respectively.

Homozygous $\mathrm{F}_{2}$ plants of a cross between a FRK1antisense line and a FRK3-RNAi (F3R1) line did not show altered growth and the plants appeared similar to WT plants (Fig. 4), suggesting that the two genes might not be expressed in the same cells or that FRK2 might be able to compensate for the decreases in both FRK1 and FRK3. However, plants homozygous for antisense suppression of both FRK1 and FRK2 showed severe growth inhibition even relative to FRK2-antisense plants with more pronounced effects on leaf wilting than those observed among FRK2-antisense plants (Fig. 4). The additive effect of FRK1 and FRK2 suppression was quite similar to the additive effect observed in plants with combined suppression of FRK2 and FRK3 (Fig. 4), which also resulted in very stunted plants that were unable to grow taller than $20 \mathrm{~cm}$ and did not flower and set fruit. These results suggest that FRK1 contributes to tomato vascular development together with FRK2.

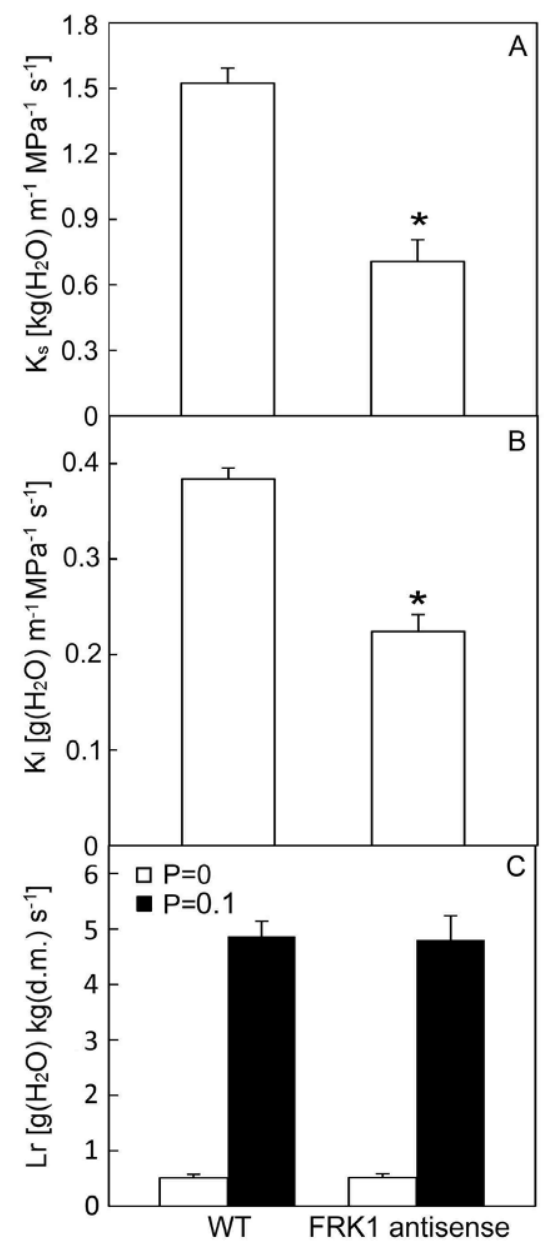

Fig. 3. The effect of SlFRK1 suppression on stem and root hydraulic conductivity. $A$ - stem hydraulic conductivity (Ks) normalized to xylem cross-sectional area; $B$ - stem hydraulic conductivity (Kl) normalized to leaf area. Means \pm SEs, $n=8$, $*$ - statistically significant differences $(P<0.05)$ between WT and FRK1-antisense plants. $C$ - Root conductivity (Lr) was determined based on pressure-driven exudation (black bars, pressure $=0.1 \mathrm{MPa}$ ) and osmotically generated flow (open bars). Means \pm SEs, $n=4$.

To better understand the impact of FRK1 on tomato vascular development, we analyzed the anatomy of the homozygote double-mutant FRK1-antisense $\times$ FRK2antisense plants. Cross-sections taken from stems showed that the width of the secondary xylem was drastically 
reduced compared to WT stems and to the stems of each of the independent parent lines (Fig. 5), indicating reduced cambial activity. In addition, the double-mutants secondary xylem contains much fewer vessels (Fig. 5D). However, since these plants are very stunted, it might be expected that they would have less secondary xylem, making it impossible to determine whether the reduced secondary xylem is the cause or the outcome of the growth inhibition.

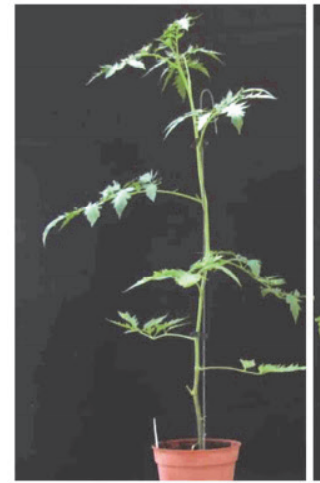

FRK1antisense

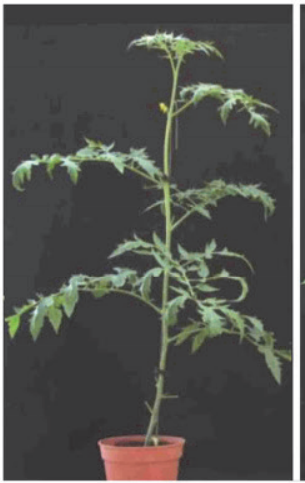

FRK3-RNAi

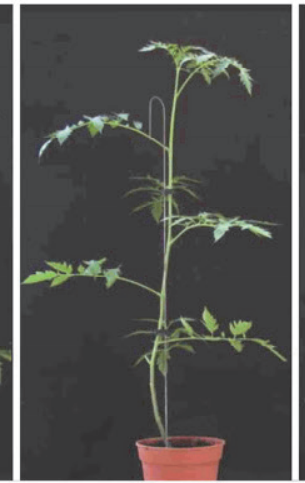

FRK1antisense $x$

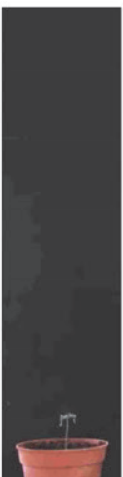
FRK2antisen $x$
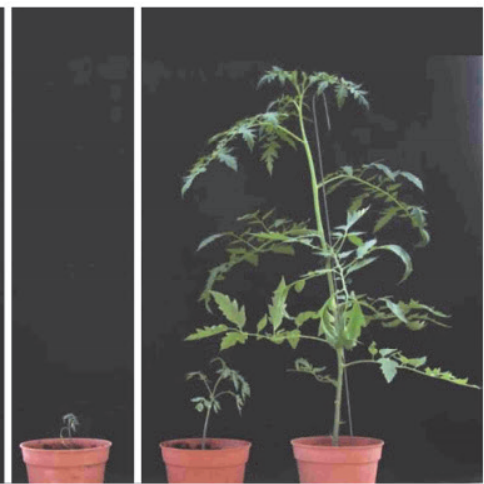

FRK1- FRK2- WT tisense antisense $x$ FRK2antisense

Fig. 4. Co-suppression of SlFRK1 and SlFRK2 enhanced growth inhibition. Plants were grown under normal conditions in the greenhouse and photographed about 10 weeks after germination.
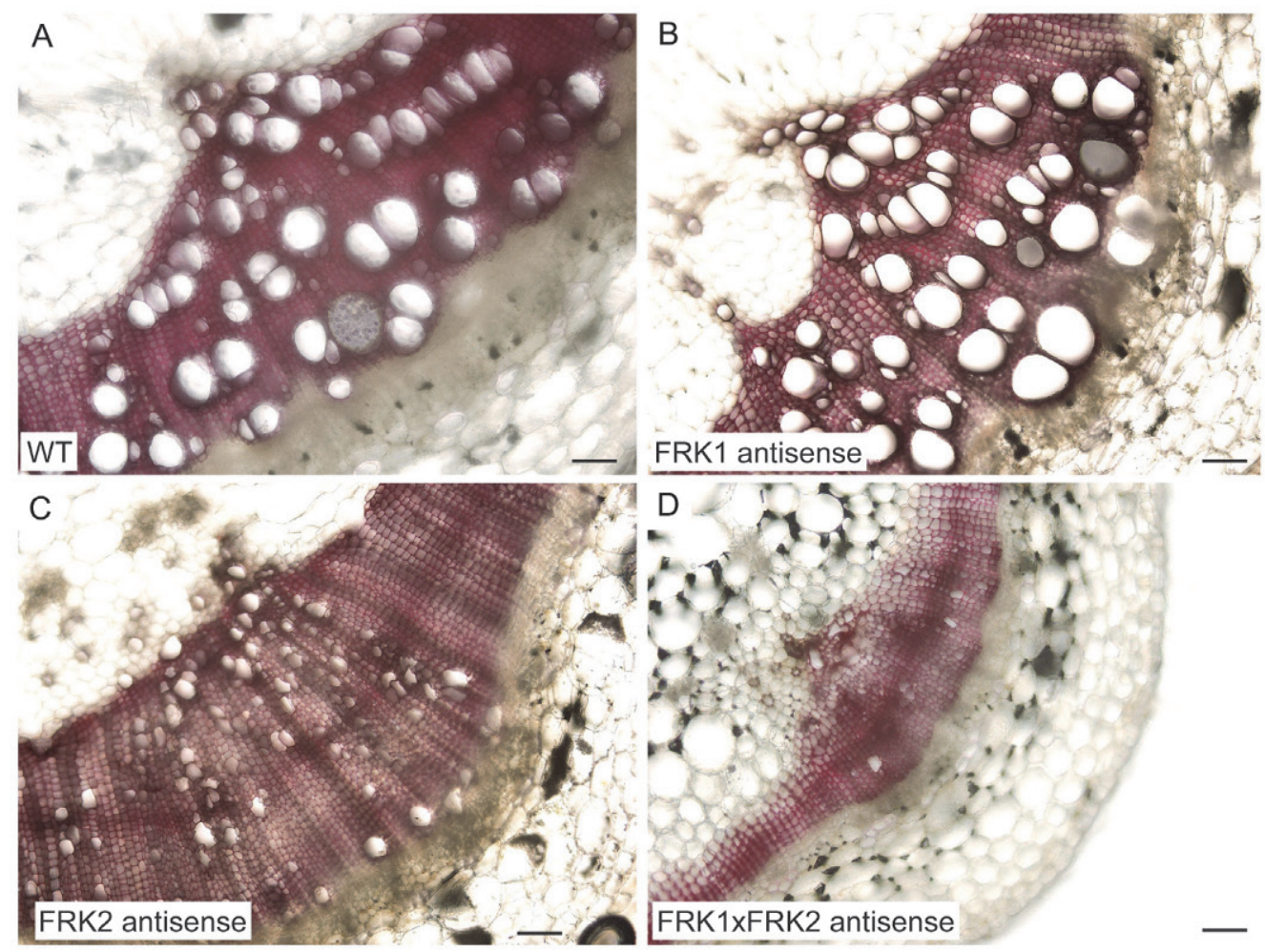

Fig. 5. Co-suppression of SIFRK1 and SIFRK2 reduced secondary xylem area. Stem cross-sections of 10-week-old plants shown in Fig. 4 taken from the internode between the first and second true leaves, showing reduced secondary xylem width (stained pink) and reduced vessel width. Scale bars $-100 \mu \mathrm{m}$.

Interestingly, phloroglucinol stained lignin in the xylem fibers, but did not in the phloem fibers in all the lines (Fig. 5), suggesting that phloem fibers are less sensitive to phloroglucinol stain, possibly due to less lignin or different lignin properties. Similar differential staining of xylem and phloem fibers has been observed in tobacco stem cross-sections (Townsley et al. 2013). Although phloroglucinol did not stain the lignin in the 
phloem fibers, cross-sections of the F2 homozygous FRK1 and FRK2 antisense plants clearly revealed that some phloem fibers had narrower cell walls and some were distorted (Fig. 4 Suppl.) suggesting SlFRK1 might be important for phloem fiber development. Distorted and thin cell walls of phloem fibers were previously observed in stems of plants of the FRK3-RNAi line (Fig. 5 Suppl.), F3R4, which had decreased expression of all three FRKs (Stein et al. 2016). However, it is not clear which of the FRKs contributed to the distorted phloem fibers in this phenotype.
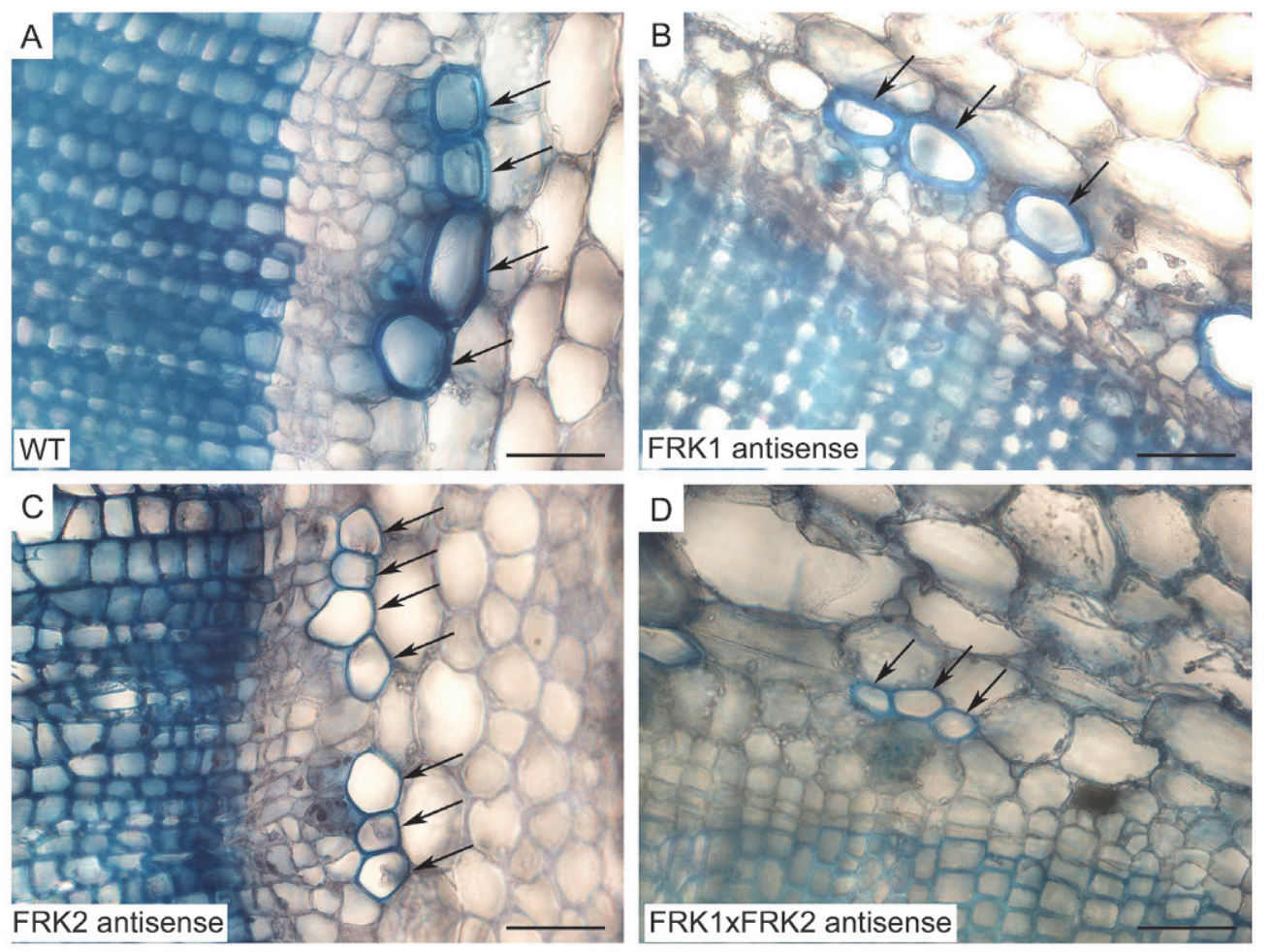

Fig. 6. Co-suppression of SlFRK1 and SlFRK2 reduced the size of phloem fiber cells. Stem cross-sections of 10-week-old plants shown in Fig. 4 taken from the internode between the first and second true leaves, showing smaller phloem fibers (arrows). Scale bars $-50 \mu \mathrm{m}$.

To better elucidate the effect on phloem fibers, we analyzed the stems using lacmoid staining that stain phloem fibers. Inspection of the phloem fibers in the FRK1-antisense $\times$ FRK2-antisense plants (Fig. 4) revealed that those plants had smaller phloem fiber cells than WT, FRK1-antisense, or FRK2-antisense plants (Fig. 6). These phloem fibers were not only smaller, but also had narrower cell walls with less lignin and, therefore, were not always stained by the lacmoid (Fig. 7D), in contrast to the phloem fibers in FRK2antisense $\times$ FRK3-RNAi plants, which were stained very well with lacmoid, to reveal thick and normal cell walls (Fig. 7C). Some variability in the effects on phloem fibers of FRK1-antisense $\times$ FRK2-antisense plant was observed, with a few phloem fibers stained with lacmoid and stained fibers usually possessing thin cell walls (Fig. $7 E, F)$. We assume that this variability is a result of the differential suppression of SlFRK1 or SlFRK2. Nevertheless, the appearance of this phenotype in the FRK1-antisense $\times$ FRK2-antisense double-mutant and not in FRK2-antisense $\times$ FRK3-RNAi plants or in FRK1antisense $\times$ FRK3-RNAi plants indicates that it is caused by the specific co-suppression of SlFRK1 and SIFRK2 and implies a specific role for FRK1 in phloem fiber development together with FRK2.

The decrease in phloem fiber cell-wall width and lignification together with no obvious effects on the surrounding cells implies reduced metabolism and the reduced ability of these cells to utilize the sugars for the synthesis of cell-wall components such as cellulose or lignin. These findings are congruent with those of a previous study that found that the FRK in aspen wood is very important for cell-wall metabolism through carbon partitioning to cellulose in xylem fibers (Roach et al. 2012).

Interestingly, unlike FRK2, FRK1 is not inhibited by fructose and its affinity for fructose is much lower than that of FRK2. It is not clear why some FRKs are inhibited by fructose while others are not. We speculate that FRK1 may complement FRK2, whose activity is inhibited by fructose, perhaps when the concentration of fructose rises in certain cells that need high levels of carbon metabolism, such as developing trachea vessels and phloem fibers that have thick cell walls. 

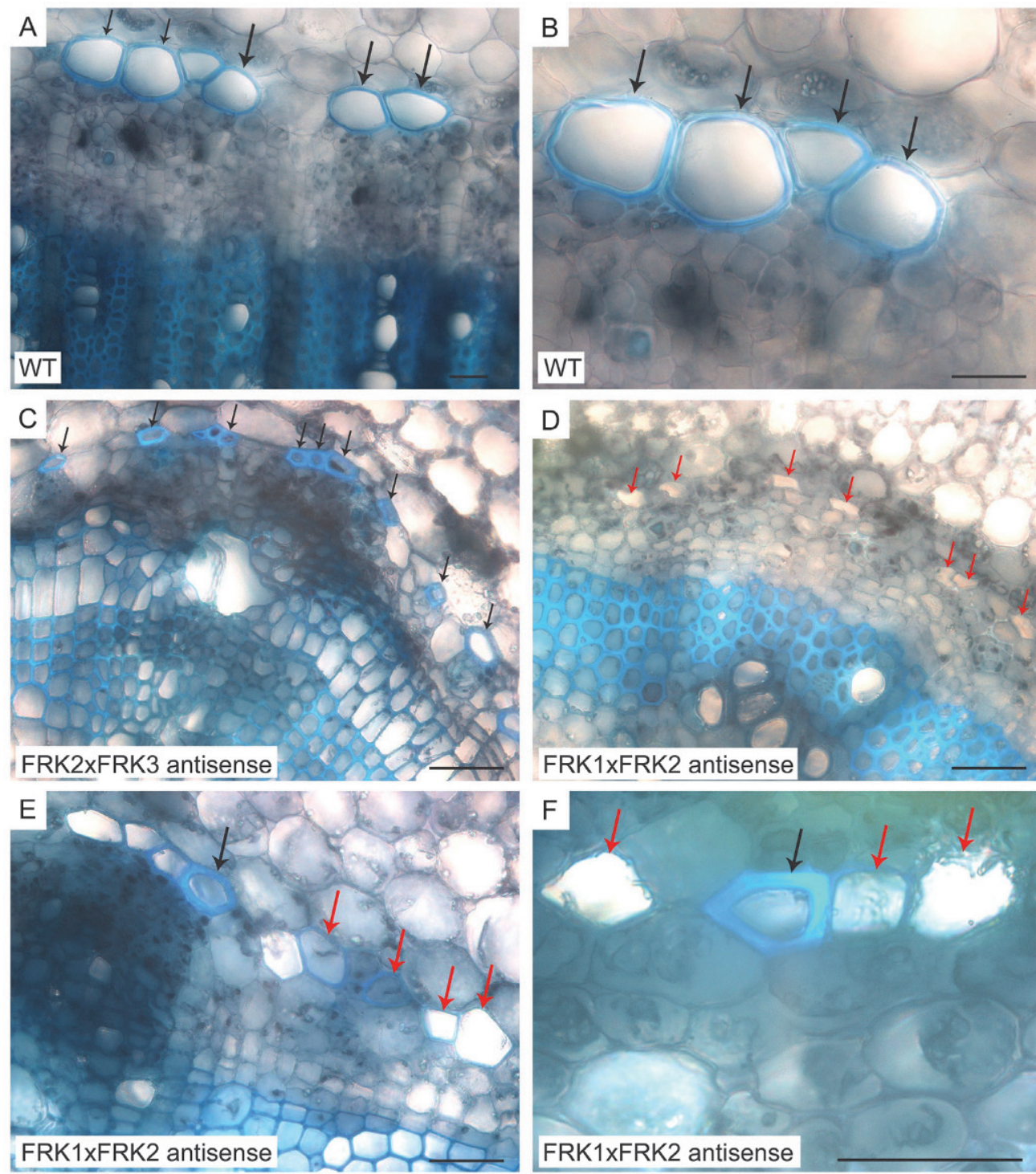

Fig. 7. Co-suppression of SlFRK1 and SlFRK2 reduced the lignification and cell-wall width of phloem fiber cells. Stem crosssections of 10-week-old plants taken from the internode between the first and second true leaves. Black arrows - normal phloem fibers; red arrows - thin cell-wall with reduced lignification in phloem fibers. Scale bars - $50 \mu \mathrm{m}$.

\section{Conclusions}

This work illustrates the importance of the cytosolic FRK1 for tomato vascular development and adds to our understanding of the roles of the other two major FRKs, the cytosolic FRK2 and the plastidic FRK3, in vascular development. The FRK1 was found to be important for water conductance through vessels and for phloem fiber

development alongside FRK2. The main effects observed after SIFRK1 suppression were reduced cell-wall width and reduced lignification resulting in distorted xylem vessels and phloem fibers, indicating that fructose phosphorylation in the vascular tissues is highly important for cell-wall metabolism.

\section{References}

Aloni, R.: Role of auxin and sucrose in the differentiation of sieve and tracheary elements in plant tissue cultures. Planta 150: 255-263, 1980.

Dai, N., Kandel, M., Petreikov, M., Levine, I., Ricard, B.,

Rothan, C., Schaffer, A.A., Granot, D.: The tomato hexokinase LeHXK1: cloning, mapping, expression pattern and phylogenetic relationships. - Plant Sci. 163: 581-590, 2002. 
Dai, N., Schaffer, A., Petreikov, M., Granot, D.: Potato (Solanum tuberosum L.) fructokinase expressed in yeast exhibits inhibition by fructose of both in vitro enzyme activity and rate of cell proliferation. - Plant Sci. 128: 191197, 1997.

Damari-Weissler, H., Kandel-Kfir, M., Gidoni, D., Mett, A., Belausov, E., Granot, D.: Evidence for intracellular spatial separation of hexokinases and fructokinases in tomato plants. - Planta 224: 1495-1502, 2006.

Damari-Weissler, H., Rachamilevitch, S., Aloni, R., German, M.A., Cohen, S., Zwieniecki, M.A., Holbrook, N.M., Granot, D.: LeFRK2 is required for phloem and xylem differentiation and the transport of both sugar and water. Planta 230: 795-805, 2009.

David-Schwartz, R., Weintraub, L., Vidavski, R., Zemach, H., Murakhovsky, L., Swartzberg, D., Granot, D.: The SIFRK4 promoter is active only during late stages of pollen and anther development. - Plant Sci. 199-200: 61-70, 2013.

Dennis, D.T., Blakeley, S.D.: Carbohydrate Metabolism. American Society of Plant Physiologists, Rockville 2000.

Gardner, A., Davies, H.V., Burch, L.R.: Purification and properties of fructokinase from developing tubers of potato (Solanum tuberosum L.). - Plant Physiol. 100: 178-183, 1992.

German, M.A., Asher, I., Petreikov, M., Dai, N., Schaffer, A.A., Granot, D.: Cloning, expression and characterization of LeFRK3, the fourth tomato (Lycopersicon esculentum Mill.) gene encoding fructokinase. - Plant Sci. 166: 285-291, 2004.

German, M.A., Dai, N., Chmelnitsky, I., Sobolev, I., Salts, Y., Barg, R., Schaffer, A.A., Granot, D.: LeFRK4, a novel tomato (Lycopersicon esculentum Mill.) fructokinase specifically expressed in stamens. - Plant Sci. 163: 607-613, 2002.

German, M.A., Dai, N., Matsevitz, T., Hanael, R., Petreikov, M., Bernstein, N., Ioffe, M., Shahak, Y., Schaffer, A.A., Granot, D.: Suppression of fructokinase encoded by LeFRK2 in tomato stem inhibits growth and causes wilting of young leaves. - Plant J. 34: 837-846, 2003.

Gorska, A., Ye, Q., Holbrook, N.M., Zwieniecki, M.A.: Nitrate control of root hydraulic properties in plants: translating local information to whole plant response. - Plant Physiol. 148: $1159-1167,2008$.

Granot, D.: Role of tomato hexose kinases. - Funct. Plant Biol. 34: 564-570, 2007.
Granot, D., David-Schwartz, R., Kelly, G.: Hexose kinases and their role in sugar-sensing and plant development. - Front. Plant Sci. 4: 44, 2013.

Hoagland, D.R., Arnon, D.I.: The water culture method for growing plants without soil. - Calif. Agr. Exp. Sta. Circular 347: 1-39, 1950.

Kanayama, Y., Dai, N., Granot, D., Petreikov, M., Schaffer, A., Bennett, A.B.: Divergent fructokinase genes are differentially expressed in tomato. - Plant Physiol. 113: 1379-1384, 1997.

Odanaka, S., Bennett, A.B., Kanayama, Y.: Distinct physiological roles of fructokinase isozymes revealed by genespecific suppression of frk1 and frk2 expression in tomato. Plant Physiol. 129: 1119-1126, 2002.

Petreikov, M., Dai, N., Granot, D., Schaffer, A.A.: Characterization of native and yeast-expressed tomato fruit fructokinase enzymes. - Phytochemistry 58: 841-847, 2001.

Renz, A., Stitt, M.: Substrate-specificity and product inhibition of different forms of fructokinases and hexokinases in developing potato tubers. - Planta 190: 166-175, 1993.

Roach, M., Gerber, L., Sandquist, D., Gorzsas, A., Hedenstrom, M., Kumar, M., Steinhauser, M.C., Feil, R., Daniel, G., Stitt, M., Sundberg, B., Niittyla, T.: Fructokinase is required for carbon partitioning to cellulose in aspen wood. - Plant $\mathrm{J}$. cell. mol. Biol. 70: 967-977, 2012.

Ruzin, S.E.: Plant Microtechnique and Microscopy. - Oxford University Press, Oxford - New York, 1999.

Stein, O., Avin-Wittenberg, T., Krahnert, I., Zemach, H., Bogol, V., Daron, O., Aloni, R., Fernie, A.R., Granot, D.: Arabidopsis fructokinases are important for seed oil accumulation and vascular development. - Front. Plant Sci. 7: 2047. doi: 10.3389/fpls.2016.02047, 2017.

Stein, O., Damari-Weissler, H., Secchi, F., Rachamilevitch, S., German, M.A., Yeselson, Y., Amir, R., Schaffer, A., Holbrook, N.M., Aloni, R., Zwieniecki, M.A., Granot, D.: The tomato plastidic fructokinase SIFRK3 plays a role in xylem development. - New Phytol. 209: 1484-1495, 2016.

Townsley, B.T., Sinha, N.R., Kang, J.: KNOX1 genes regulate lignin deposition and composition in monocots and dicots. Front. Plant Sci. 4: 121, 2013

Weise, A., Lalonde, S., Kuhn, C., Frommer, W.B., Ward, J.M.: Introns control expression of sucrose transporter LeSUT1 in trichomes, companion cells and in guard cells. - Plant mol. Biol. 68: 251-262, 2008 